\title{
THE HIGH LUMINOSITY PERFORMANCE OF CESR WITH THE NEW GENERATION SUPERCONDUCTING CAVITY *
}

\author{
$\underline{\text { S. Belomestnykh }}{ }^{\#}$, Laboratory of Nuclear Studies, Cornell University, Ithaca, NY 14853, U.S.A.
}

\section{Abstract}

With the installation of the first SRF cavity in September of 1997, the upgrade of the CESR RF system has begun [1]. This cavity belongs to the new generation of socalled HOM damped cavities designed for high current storage rings [2]. The upgrade is proceeding by replacing one by one old copper cavities with superconducting ones during short machine shutdowns.

CESR continues to demonstrate spectacular performance. It operates with 36 bunches in 9 trains of 4 bunches in each beam with total beam current up to 550 $\mathrm{mA}$ at the start of fills for high energy physics. In the course of last year both peak luminosity and integrated luminosity per day increased by $67 \%$ and are equal now to $8.0 \times 10^{32} \mathrm{~cm}^{-2} \mathrm{~s}^{-1}$, and $40.2 \mathrm{pb}^{-1} /$ day. The latter number corresponds roughly to 43 thousands of $B$ mesons per day. The first superconducting cavity has been in operation in CESR since October of 1997. The cavity operates at accelerating gradients up to $7 \mathrm{MV} / \mathrm{m}$ and delivers to the beam maximum RF power of $220 \mathrm{~kW}$ through the ceramic window, the record value for superconducting cavities. We found that pulsed power processing without beam is extremely helpful in improving RF window performance. The maximum HOM power extracted from the beam by one cavity is $5.7 \mathrm{~kW}$.

Four single-cell HOM damped cavities will eventually support a total beam current of $1 \mathrm{~A}$. Because of the very low impedance of superconducting cavity module, we calculate the longitudinal instability threshold from cavity HOMs to be in excess of $1 \mathrm{~A}$. The second cavity was installed in CESR during October'98 shutdown. The third cavity is already installed in CESR, and its commissioning will start immediately after this conference. The fourth cryomodule is being assembled and is schedule for installation in summer of 1999.

\section{INTRODUCTION}

Prior to the installation of the first SRF cavity, since November of 1994, CESR operated in the Phase II configuration with nine two-bunch trains and $\pm 2.1 \mathrm{mrad}$ crossing angle [3]. The maximum total current in two colliding beams was $350 \mathrm{~mA}$, peak luminosity up to $4.4 \times 10^{32} \mathrm{~cm}^{-2} \mathrm{~s}^{-1}$, and maximum integrated luminosity of $23.6 \mathrm{pb}^{-1} /$ day. Total current was limited by a longitudinal coupled bunch instability caused by higherorder modes in the 5-cell copper RF accelerating cavities $[4,5]$.

\footnotetext{
"Work supported by the National Science Foundation.

"Email: sab@1ns62.lns.cornell.edu
}

Table 1: CESR Phase III Parameters.

\begin{tabular}{|l|c|}
\hline Beam energy & $5.289 \mathrm{GeV}$ \\
Number SRF cavities & 4 \\
Total RF voltage & $7.2-12 \mathrm{MV}$ \\
Natural bunch length & $13 \mathrm{~mm}$ \\
Number of bunch trains & 9 \\
Number of bunched per train & 5 \\
Bunch spacing & $14 \mathrm{~ns}$ \\
Total beam current in two & $1 \mathrm{~A}$ \\
beams & $2.7 \mathrm{mrad}$ \\
Crossing angle & 0.04 \\
Vertical tune shift parameter & $1.7 \times 10^{33} \mathrm{~cm}^{-2} \mathrm{~s}^{-1}$ \\
Luminosity & \\
\hline
\end{tabular}

Phase III of the CESR luminosity upgrade involves replacement of the final focus quadrupoles and interaction region vacuum chambers, and installation of the new superconducting RF system [6]. The CESR III design parameters are shown in Table 1. To achieve the desired luminosity of $1.7 \times 10^{33} \mathrm{~cm}^{-2} \mathrm{~s}^{-1}, 1$ Ampere beam current must be stored in multibunch beams in CESR. Due to such a high beam currents, input couplers, windows, and HOM loads of accelerating cavities must be capable to deal with very high RF power. The big number of bunches puts severe restriction on the $Q$ factors and $R / Q$ s of HOMs in order to avoid multibunch instabilities. Apart from this, even fundamental mode can cause problems if its $R / Q$ is too high [7, 8, 9]. Minimizing the number of cavities in the ring helps reducing total impedance of RF system as well. These challenges to CESR RF system are similar to challenges to RF systems of other high current machines and were reviewed at previous conferences [10, 11, 7, 12, 2]. Several superconducting (SC) and normal conducting (NC) cavity designs have been developed to meet new requirements of high power and low impedance. A storage ring RF system based on SC cavities has lower impedance than its $\mathrm{NC}$ counterpart because of i) the higher achievable accelerating gradient decreases the number of cells needed, ii) low $R / Q$ of the fundamental mode, iii) simple and effective method of HOM damping. These factors and a long experience with superconducting RF (SRF) at Cornell determined the cavity choice for the CESR Upgrade.

\section{CESR RF SYSTEM UPGRADE}

Table 2 contains the major design parameters of the CESR Phase III RF system [13]. The system consists of 
four single cell superconducting niobium cavities [14] in their individual cryostats. The cavities are located in the CESR East (cavities E1 and E2) and West (cavities W1 and W2) RF straight sections. Two cavities are fed by $\mathrm{RF}$ power from one klystron via magic T. The HOM power absorbed by ferrite loads is expected to be 13.7 $\mathrm{kW}$ per cavity. With installation of all four SRF cavities the bunch length in CESR can be reduced to $13 \mathrm{~mm}$. RF power of $325 \mathrm{~kW}$ will be delivered to the beam through each input coupler and RF window. New refrigeration and cryogen distribution system is installed to provide liquid helium to RF cavities, interaction point superconducting quadrupole lenses and superconducting solenoid of detector CLEO. As part of the Phase III of the CESR Luminosity Upgrade Program, other components of RF system are being upgraded as well. $800 \mathrm{~kW}$ klystrons will replace old $600 \mathrm{~kW}$ ones. Two new 2 MW SLAC-type high voltage klystron power supplies are installed.

Table 2: Parameters of the CESR III RF system.

\begin{tabular}{|l|c|}
\hline Frequency & $499.765 \mathrm{MHz}$ \\
Accelerating field & $6-10 \mathrm{MV} / \mathrm{m}$ \\
Effective cell length & $0.3 \mathrm{~m}$ \\
Number of cavities & 4 \\
Total RF voltage & $7.2-12 \mathrm{MV}$ \\
Cryomodule length & $2.86 \mathrm{~m}$ \\
$\mathrm{R} / \mathrm{Q}\left(\mathrm{R}=\mathrm{V}^{2} / \mathrm{P}\right)$ & $89 \mathrm{ohm}$ \\
$\mathrm{Q}_{0}$ at operating field $(4.5 \mathrm{~K})$ & $>10^{9}$ \\
$\mathrm{Q}_{\text {ext }}$ of $\mathrm{RF}$ coupler & $2 \times 10^{5}$ \\
Cryostat static heat losses & $30 \mathrm{~W}$ \\
$\begin{array}{l}\text { Cryostat liquid He volume } \\
\text { Loss factor of a module with } \\
\text { one taper at } \sigma_{\mathrm{z}}=13 \text { mm }\end{array}$ & 520 liters \\
$\begin{array}{l}\text { Power delivered to } 1 \text { A beam } \\
\text { through the RF window }\end{array}$ & $0.48 \mathrm{~V} / \mathrm{pC}$ \\
\hline
\end{tabular}

\section{CRYOMODULE}

A novel superconducting cavity geometry [14] was initially proposed for the Cornell B-factory project. Later on this cavity became a part of the Phase III of the CESR luminosity upgrade program. Initial R\&D efforts $[15,16,17,18]$ were followed by the development and tests of the cryomodule-prototype and its elements [19]. A successful beam test in 1994 [20] allowed us to design new, MARK II, cryostat to meet rather tight requirements of the CESR tunnel [21].

A drawing of the cryomodule is presented in Figure 1. A $500 \mathrm{MHz}$ niobium cavity is placed inside the helium vessel. Bell-shaped cavity geometry with wide openings of $24 \mathrm{~cm}$ diameter beam tubes makes $R / Q$ of the HOMs small in comparison with a conventional cavity geometry (Figure 2) and allows monopole HOMs to propagate toward a ferrite absorbers. The $Q$ factors of most higher-order modes are of the order of 100.
Because the first two dipole modes have resonant frequencies below cut-off of the $24 \mathrm{~cm}$ diameter round beam tube (RBT), beam tube on one side has flutes which lower the cut-off frequency. As a result, these dipole HOMs can now propagate via fluted beam tube (FBT) to the FBT side load.

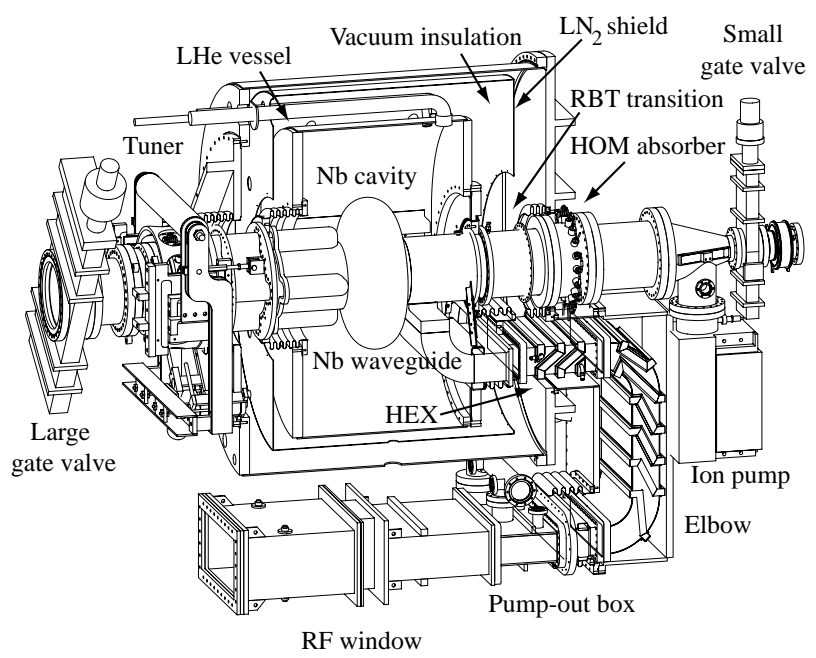

Figure 1. The CESR B-cell cryomodule.

Turning to the cryostat, the helium vessel inside surface is covered with copper foil to make its temperature more even during cool down. The He vessel is suspended inside the cryostat on four invar rods. The space between the He vessel and cryostat walls serves as vacuum insulation. Also, it contains liquid nitrogen cooled radiation shield, 60 layers of mylar super insulation, and magnetic shielding. Within vacuum envelope of the cryostat are thermal transition pieces on the RBT and FBT beam pipes and the waveguide designed to keep radiation and conduction heat loads of the liquid He bath at low level. The beam line transition pieces are of the same cross section as the ends of the niobium cavity. They are made of $1 \mathrm{~mm}$ thick stainless steel with $3.8 \mu \mathrm{m}$ copper coating to reduce RF losses due to beam current. The waveguide thermal transition incorporates a gaseous helium heat exchanger (HEX) to help carry away the heat generated in the walls by the high RF power. This stainless steel unit is plated inside with $25 \mu \mathrm{m}$ of copper. The next portion of the waveguide, the $180^{\circ}$ waveguide $\mathrm{U}$, is cooled by liquid nitrogen.

The ceramic RF window is connected to the waveguide inside the cryostat via the short section furnished with two $60 \mathrm{l} / \mathrm{s}$ ion pumps. The window is capable to transfer RF power up to $500 \mathrm{~kW}$ in travelling mode [22].

In both directions along the beam line outside the cryostat are the ferrite-lined higher-order mode loads $[23,24]$. They serve to absorb the beam induced HOM power. 


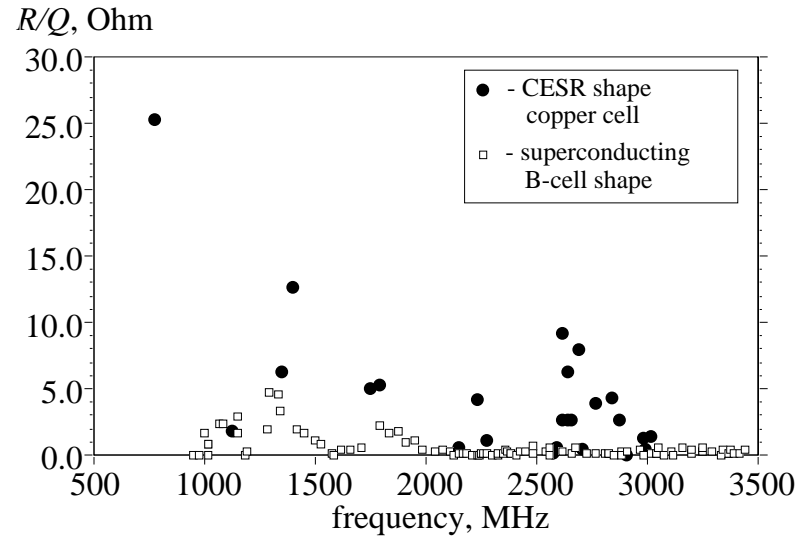

Figure 2. Comparison of HOM $R / Q$ s of the conventional copper cavity and superconducting B-cell cavity.

The cryomodules are designed for installation alone or in a pair. Standard installation in CESR is in pairs. RBT end of the module is connected to the CESR beam pipe. It is equipped with a taper from $24 \mathrm{~cm}$ diameter of the cavity beam pipes to a smaller size cross section of adjacent CESR vacuum chamber. A small gate valve serves to separate cavity vacuum from machine vacuum whenever it is necessary. Two cryomodules in a pair are connected to each other by FBT ends with $24 \mathrm{~cm}$ diameter straight vacuum pipe. Vacuum volumes of two cryomodules can be separated by large diameter gate valves. In case of one cavity installation a taper is connected to the FBT end of the cryomodule next to the large gate valve.

\section{SRF CAVITY INSTALLATION AND COMMISSIONING RESULTS}

The first SRF cavity was installed in CESR in September of 1997 in E2 location. Using a NC and a SC cavity as a pair makes operation somewhat complicated. To maintain the same RF field as the copper cavity, the SRF cavity requires much less incident power without beam present [25]. For example, to get accelerating voltage of 1.5 MV, one needs to provide forward power of approximately $100 \mathrm{~kW}$ to the NRF cavity, but only about $30 \mathrm{~kW}$ to the SRF cavity with nearly all of it reflected. The numbers converge with increasing beam current, but even at 0.6 A total beam current we get forward power of $250 \mathrm{~kW}$ for the NRF cavity versus $210 \mathrm{~kW}$ for the SRF cavity. In order to keep voltages reasonably even, it was proposed [25] to use a waveguide $4 \mathrm{~dB}$ hybrid instead of magic $T$ as an $R F$ power splitter. In the $4 \mathrm{~dB}$ splitter configuration the SRF cavity was operated at a field of $6.3 \mathrm{MV} / \mathrm{m}$ or voltage of $1.9 \mathrm{MV}$.

At first, multipacting and arcing in the RF coupler region limited the CESR beam current and delivered by the SRF cavity beam power at about $100 \mathrm{~kW}$. After exploring several RF processing techniques, we found that the best results are achieved when processing without beam by pulsed power on or close to cavity resonance. By pulse processing, the beam power could gradually be raised to $140 \mathrm{~kW}$ and total beam current to $350 \mathrm{~mA}$. At this power level we observed for a first time dependence of the RF power on the cavity field: transmitted power is higher when there is bigger standing wave component. Computer simulations [26] confirmed that travelling to standing wave mixing ratio affects multipacting bands.

Also, we observed fast vacuum events caused by releasing cryopumped hydrogen due to RF heating of the HEX surface, and following arc. Analysis of residual gas evolution [27] during cavity warm up showed that after about 2 months of operation without warming the cavity up, cold surfaces accumulated several equivalent monolayers of hydrogen. Eventually it was found that baking $\mathrm{RF}$ window ceramics to $>150^{\circ} \mathrm{C}$ and periodic warm ups cures this effect.

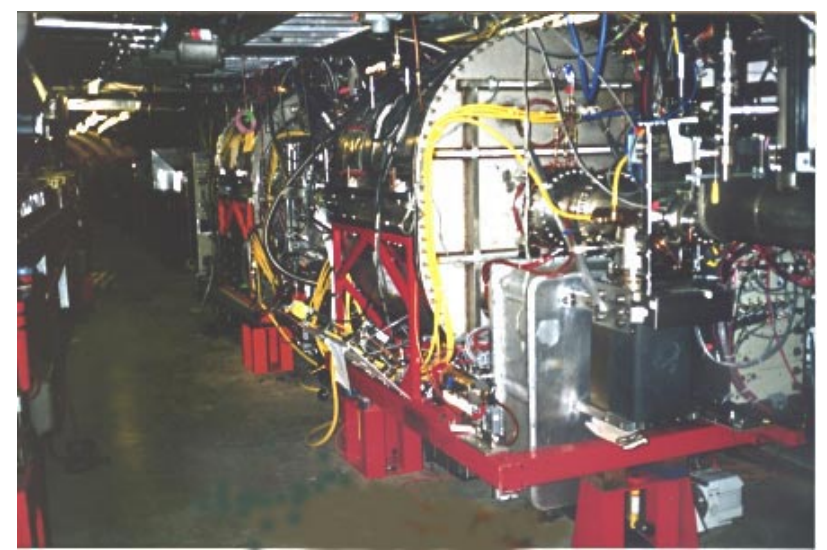

Figure 3. Photo of E1 and E2 cryomodules in the CESR tunnel.

To explore the ability of E2 SRF cavity to deliver higher RF powers to beam, we switched from a $4 \mathrm{~dB}$ hybrid back to a magic T $3 \mathrm{~dB}$ waveguide configuration after the next cavity warm up. This allowed us to load the SRF cavity stronger with beam and reached $212 \mathrm{~kW}$ in travelling wave and to operate at the field level of 7 $\mathrm{MV} / \mathrm{m}$.

A low frequency vibration from the neighbouring NRF cavity cooling water pump caused microphonics effect in the E2 cavity. Added stiffeners reduced this effect to a tolerable level. This effect disappeared when the NRF cavity was replaced with the second superconducting cavity.

Operating experience obtained with the first SRF cavity allowed us to introduce several changes in cryomodule design and preparation procedure [1]. As we will see in the next section, as we addressed these problems, CESR continued to set new peak and integrated luminosity records. 


\section{CESR PERFORMANCE AND LIMITATIONS}

With installation of the first SRF cavity we were able to raise beam current steadily. Initial beam current limitation due to RF window multipacting was overcome using pulse processing. As it was mentioned earlier, the major limitation of the total beam current prior to installation of the E2 cavity was the longitudinal coupled bunch instability. The instability current threshold measurements [4] and computer calculations [28] showed that this threshold depends on the bunch spacing within the bunch train. The CESR standard filling patterns allow the bunches within each train to be spaced in increments of $14 \mathrm{~ns}$, up to maximum of 5 bunches per train. Initially CESR was operating in $9 \times 2$ mode with 28 ns spacing. After learning that $42 \mathrm{~ns}$ spacing had a higher current threshold, the routine operation of CESR was changed to this bunch spacing. Nevertheless, this was not enough as the single beam threshold current was only about $230 \mathrm{~mA}$. A longitudinal multibunch feedback system has been implemented [29] based on a digital signal processing to produce bunch by bunch beam stabilization.

In spite of installation of a low impedance SC cavity, the total HOM impedance of the ring was still dominated by remaining $\mathrm{NC}$ cavities and changes of the beam current thresholds were small [30]. Moreover, the current threshold at $42 \mathrm{~ns}$ spacing became lower. The instability growth rate calculations showed that as long as there is even one $\mathrm{NC}$ cavity in the ring, one should not expect a dramatic change in the current threshold. Therefore, it has become crucial to have detail information about current threshold for all possible bunch train patterns and choose the bunch pattern appropriately. That is why CESR operation has been switched from $9 \times 2$ to $9 \times 3$ configuration, and later to $9 \times 4$ bunch train configuration. Latest measurements of instability thresholds and HOM spectra of horizontal separators indicate that there could be other sources of a narrow band impedance apart from RF cavities [31]. This impedance might limit the threshold current after complete installation of the new SRF system and it is important to have reliable longitudinal feedback system in place.

During the running period with one SRF cavity the maximum extracted $\mathrm{HOM}$ power was about $5.7 \mathrm{~kW}$ at $510 \mathrm{~mA}$ total current in two beams of nine three-bunch trains each (obtained during machine studies experiment).

E1 superconducting cavity was installed in the machine in October of 1999. Shortly after its commissioning we were able to reach and exceed 200 $\mathrm{kW}$ power delivered to beam by one cavity [1]. Operating with two SRF cavities proved to be easier and more stable than with one SRF and one NRF cavity in the same pair.

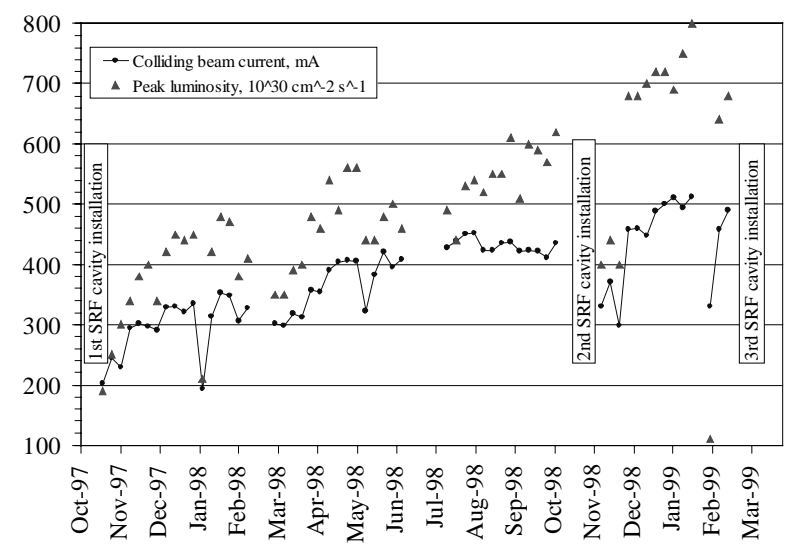

Figure 4. CESR peak luminosity and colliding beam current performance since the beginning of RF system upgrade.

The ability of SRF cavities to deliver high beam power and continuing efforts to improve performance of the longitudinal feedback system helped to increase total beam current to $550 \mathrm{~mA}$ at the beginning of the HEP fills and peak luminosity to $8 \times 10^{32} \mathrm{~cm}^{-2} \mathrm{~s}^{-1}$. Figure 4 illustrates change of the beam current brought into collision and peak luminosity week by week since the beginning of the CESR RF system upgrade. Another important factor in achieving record peak luminosity is ability to reach high beam-beam tune shift parameter $\left(\xi_{v}\right)$. In last several weeks of operation CESR was running with $\xi_{v}$ up to 0.049 . This can be attributed in part to improved alignment of magnetic elements [32]. More details about CESR performance and upgrade status can be found in [33]. Table 3 lists highest parameters achieved by CESR II.

\section{SUMMARY}

Today CESR is the highest luminosity collider running at $8 \times 10^{32} \mathrm{~cm}^{-2} \mathrm{~s}^{-1}$ with an upgrade in progress to double the luminosity. Two SC cavities have been successfully commissioned and are in operation. The SRF cavities provided reliable operation with high beam currents up to $550 \mathrm{~mA}$ and allowed CESR to establish several peak and integrated luminosity records.

The ultimate measure of CESR performance is an integrated luminosity delivered to CLEO detector. February of 1999 was the last month when CESR II was running for HEP experiments as CLEO has begun its upgrade to CLEO III. The history of monthly integrated luminosity delivered by CESR since the beginning of its Phase II upgrade is shown in Figure 5. The total luminosity delivered by the collider is $18.07 \mathrm{fb}^{-1}$.

The next one and a half month long run of CESR will be dedicated exclusively to experiments on synchrotron radiation facility CHESS and machine studies with three $\mathrm{SC}$ cavities. The third cryomodule is installed in CESR in place of remaining two NC cavities and it is being 


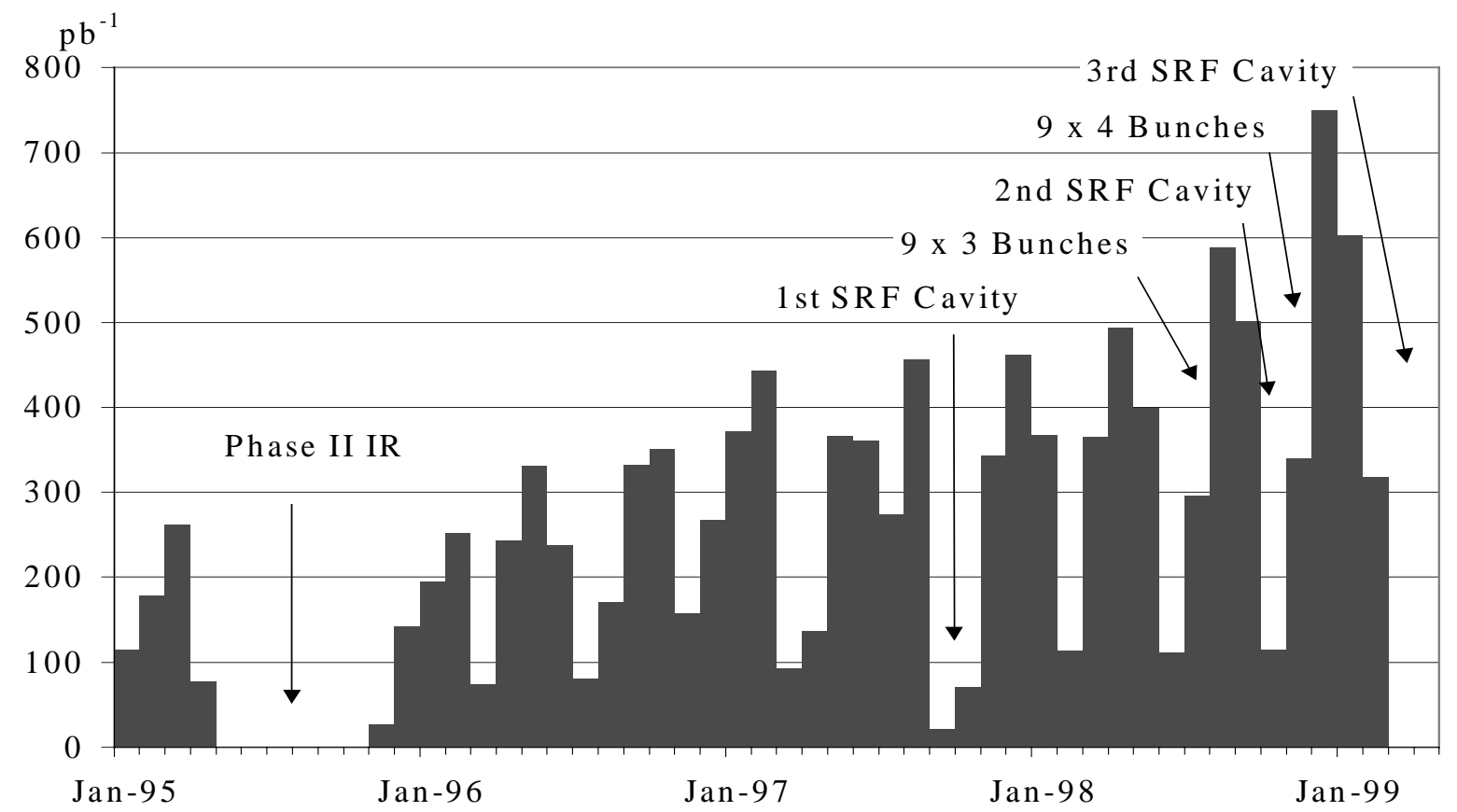

Figure 5. CESR integrated luminosity per month.

commissioned now. Three SC cavities will provide enough RF voltage and power to support two beams. The fourth cryomodule is being assembled and is scheduled for installation during long CLEO III / CESR III shutdown in summer'99. Installation of all four SRF cavities will reduce the ring impedance and provide a stable operation at high beam currents up to 1 ampere.

Table 3: Highest parameters achieved during CESR Phase II HEP performance.

\begin{tabular}{|l|c|}
\hline Peak luminosity & $8 \times 10^{32} \mathrm{~cm}^{-2} \mathrm{~s}^{-1}$ \\
Integrated luminosity per day & $40.2 \mathrm{pb}^{-1}$ \\
Integrated luminosity per month & $750 \mathrm{pb}^{-1}$ \\
Integrated luminosity per year & $4442 \mathrm{pb}^{-1}$ \\
Total current & $550 \mathrm{~mA}$ \\
Beam-beam parameter, $\xi_{v}$ & 0.050 \\
\hline
\end{tabular}

\section{ACKNOWLEDGEMENTS}

It is a pleasure for me to acknowledge that results presented here were obtained by a collective team effort of all people of the CESR SRF group and CESR operations group.

\section{REFERENCES}

[1] S. Belomestnykh, et al., MOP135, these proceedings.

[2] H. Padamsee, Proc. 6th EPAC, Stockholm, 1998.

[3] D. L. Rubin, Proc. 1997 Part. Accel. Conf., Vol. 1, p. 372.

[4] M. Billing, Proc. 1997 Part. Accel. Conf., Vol. 2, p. 2317.

[5] A. Temnykh, et al., Proc. 1997 Part. Accel. Conf., Vol. 2, p. 1718.

[6] D. L. Rubin, Proc. 6th EPAC, Stockholm, 1998.

[7] K. Akai, Proc. 5th European Part. Accel. Conf., Vol. 1, p. 205.

[8] S. Belomestnykh, Cornell LNS Report SRF970314-01 (1997).

[9] D. Fromowitz, Cornell LNS Report CON97-10.

[10] J. Kirchgessner, Part. Accel., 46(1), p. 151 (1994).

[11] J. Kirchgessner, Proc. 1995 Part. Accel. Conf., Vol. 3, p. 1469.

[12] R. Boni, Proc. 5th European Part. Accel. Conf., Vol. 1, p. 182.

[13] S. Belomestnykh, et al., Proc. 5th EPAC, Vol. 3, p. 2100.

[14] H. Padamsee, et al., Part. Accel., 40, p. 17 (1992).

[15] D. Moffat, et al., Proc. 1993 Part. Accel. Conf., Vol. 2, p. 763.

[16] D. Moffat, et al., Proc. 1993 Part. Accel. Conf., Vol. 2, p. 977.

[17] D. Metzger, et al., Proc. 1993 Part. Accel. Conf., Vol. 2, p. 1399.

[18] E. Nordberg, et al., Proc. 1993 Part. Accel. Conf., Vol. 2, p. 995.

[19] H. Padamsee, et al., Proc. 4th EPAC, Vol. 3, p. 2048.

[20] H. Padamsee, et al., Proc. of the 1995 PAC, Vol. 3, p. 1515.

[21] J. Kirchgessner, et al., Proc. 7th SRF Workshop, Vol. 1, p. 35.

[22] E. Chojnacki, et al., Part. Accel., 61, p. [309]/45 (1998).

[23] S. Belomestnykh, et al., Proc. 1995 PAC, Vol. 5, p. 3394.

[24] E. Chojnacki, W. J. Alton, MOP77, these proceedings.

[25] S. Belomestnykh, et al., Cornell LNS Report SRF961217-04.

[26] R. L. Geng and H. Padamsee, THAL5, these proceedings.

[27] R. L. Geng and H. Padamsee, MOP136, these proceedings.

[28] S. Belomestnykh, unpublished.

[29] J. Sikora, et al., TUA19, these proceedings.

[30] M. G. Billing and S. Belomestnykh, TUA18, these proceedings.

[31] A. Temnykh, private communication.

[32] R. Holtzapple and D. Rice, THP7, these proceedings.

[33] S. B. Peck, D. L. Rubin, WEAR3, these proceedings. 